\title{
Stability analysis of sliding-grazing phenomenon in dry-friction oscillator using TS fuzzy approach
}

\author{
Kamyar Mehran \\ School of Engineering \\ University of Warwick \\ Coventry, CV4 7LW, UK \\ Email: k.mehran@warwick.ac.uk \\ Bashar Zahawi \\ Department of Electrical and Computer Engineering \\ Khalifa University \\ PO Box 127788, Abu Dhabi, UAE \\ Email: bashar.zahawi@kustar.ac.ae \\ Damian Giaouris \\ Chemical Process Engineering Research Institute \\ Centre for Research and Technology Hellas \\ Thermi-Thessaloniki, Greece \\ Email: giaouris@cperi.certh.gr \\ Jihong Wang \\ School of Engineering \\ University of Warwick \\ Coventry, CV4 7LW, UK \\ Email: jihong.wang@warwick.ac.uk
}

\begin{abstract}
Dry-friction oscillators are mechanical systems with dry friction and stick-slip vibrations. In the context of control theory, the stability analysis of this type of dynamical systems is important since they exhibit non-smooth bifurcations, or most famously a sliding-grazing bifurcation inducing abrupt chaos. This paper develops a Lyapunovbased framework to study the so-called structural stability of the system, predicting the onset of such unique bifurcations. To achieve this, the non-linear system is first represented as a non-smooth Takagi-Sugeno (TS) fuzzy model, and the structural stability is then formulated as Linear Matrix Inequalities (LMI) feasibility problems with less conservative formulation. Solving the resulting LMI problem, the onset of sliding-grazing bifurcation can be accurately predicted.
\end{abstract}

Keywords: Stability Analysis, Grazing, Dry Friction, Fuzzy Logic

\section{Introduction}

Oscillator mechanisms with stick-slip motion and friction have extensive industrial applications like pneumatic actuators used for chopping and robotic pick-and-place operations. In the field of Dynamical systems and Control theory, oscillators with dry friction are classified as sliding Filippov systems [1,2], hybrid systems with discontinuous vector fields around 
the sliding region. A variety of smooth dynamics have been observed and studied in these forced vibrating systems with dry friction, including period-doubling bifurcations and intermittency leading to chaos [3-5]. More complex dynamics induced by discontinuous transitions in the sliding dynamics are also observed in this so-called non-smooth systems such as the occurrence of abrupt chaos from a stable periodic orbit upon varying a system parameter [6-8]. Stability analysis and control of this type of discontinuity-induced bifurcations (DIBs) $[9,10]$ are quite difficult. This is because existing tools for studying the stability of equilibria cannot be applied to cases where the stability of a periodic orbit due to discrete switching, i.e. structural stability, is the matter of interest $[11,12]$. The conventional analytical tool for studying DIBs in non-smooth systems is discontinuity mapping (DM) $[9,13]$. This technique is normally applied to sliding bifurcations in Filippov systems where the system trajectories can slide along the attracting portion of a switching manifold (where the vector fields becomes discontinuous) by introducing a small perturbation to the initial condition away from the trajectory of interest and deriving a local piecewise-smooth Poincaré map. However, in studying the onset of the grazing bifurcation, DM cannot be employed because of the well-known problem of the square-root singularity of the Jacobian of the Poincare map close to the grazing event [9, 14-17]. The case of sliding-grazing bifurcations in oscillators with dry friction is one of the four codimension-one bifurcation scenarios involving collision of a part of the periodic orbit with the boundary of the sliding region [9,18] [19]. The sliding-grazing bifurcation is unique since grazing and sliding scenarios occur simultaneously with respect to the switching manifold, and the resulting discontinuity map is neither continuous nor differentiable at the grazing point [2]. Therefore the construction of DM, due to the sliding flow, leads to such analytically cumbersome and computationally expensive expressions that the continuation of periodic orbits and the analysis of their sliding bifurcations demand special software using high-order algorithms. What's more, the direct numerical simulation of Filippov systems and the accurate switching between sliding and non-sliding motions is extremely difficult using existing smooth solvers [9, 20].

To overcome these difficulties, a non-smooth TS fuzzy model-based approach ${ }^{1}$ is proposed in this paper as an alternative to DM for the stability analysis of sliding-grazing orbits. The authors have already applied the TS fuzzy approach for the prediction of the onset of grazing bifurcations in mechanical hard-impact oscillators, successfully avoiding the squareroot singularity problem [17] and for the analysis and control of DIBs in switching power electronic converters (non-sliding Fillipov systems) [21]. To construct the analysis of sliding-grazing bifurcation in this paper, first it will be shown that the proposed non-smooth TS fuzzy formalism can well represent all the essential non-linearities induced by velocity and acceleration discontinuous dynamics. This is in contrast to conventional TS fuzzy modelling developed for the model-based control of smooth dynamical systems [22,23] which is unable to represent such discontinuities. A non-smooth Lyapunov framework is then developed such that the bifurcation analysis is formulated based on searching non-smooth Lyapunov functions in multiple yet flexible fuzzy operating regions and the resulting stability conditions are formulated as a Linear Matrix Inequality (LMI) problem. The resulting LMI stabilization problem, once solved by interior-point convex optimization methods [23-26], can pinpoint the onset of the abrupt chaotic orbit in sliding-grazing bifurcation. The proposed routine for the automation of flexible region partitioning plays a pivotal role in the accurate prediction of the bifurcation point.

\footnotetext{
${ }^{1}$ We refer to the proposed TS fuzzy model able to represent a non-smooth system as a non-smooth TS fuzzy model.
} 


\section{Modelling of non-smooth dry-friction oscillator}

\subsection{Dry-friction oscillator}

The model that we are studying here is a forced, one-degree-of-freedom oscillator with dry friction and stick-slip vibrations [27], illustrated in Fig. 1. This model consists of a block which can move on a single horizontal surface, with mass $M$, that is attached to a fixed point through a spring with a stiffness coefficient of $K$ and damping coefficient $c$. The block is subject to a sinusoidal forcing and placed on a rough driving belt moving with a constant velocity $v_{\text {con }}$ (which without loss of generality can be scaled to unity) such that the relative motion between the block and belt can be closely approximated by a kinematic dry-friction law. Therefore, the corresponding equation of motions can be expressed as

$$
M \ddot{u}+c \dot{u}+K u=P(1-\dot{u})+F \cos (\varpi t)
$$

Under non-dimensionalization, the mass $M$ of the block and stiffness coefficient $K$ can all be scaled to 1 and dry-friction element $c$ can be set to 0 as its contribution to damping is very small. The resulting equations of motion can then be expressed in dimensionless form as

$$
\ddot{u}+u=P(1-\dot{u})+F \cos (\varpi t)
$$

where the non-linear Coulomb friction law is described by

$$
P(1-\dot{u})=\alpha_{0} \operatorname{sgn}(1-\dot{u})-\alpha_{1}(1-\dot{u})+\alpha_{2}(1-\dot{u})^{3}
$$

The term $(1-\dot{u})$ represents the relative velocity between the driving belt and the moving block. In the case when $\dot{u}=1$, the relative velocity is 0 and the kinematic friction is set-valued, i.e., $-\alpha_{0}<P(1-\dot{u})<\alpha_{0}$. The coefficients of the kinematic friction characteristic $\alpha_{0}, \alpha_{1}$ and $\alpha_{2}$ are determined by the material characteristics of the block and the belt. Following the original study by Yoshitake and Sueoka [27], we shall take the values $\alpha_{0}=\alpha_{1}=1.5$ and $\alpha_{2}=0.45$. $F$ and $\varpi$ are the amplitude and frequency of sinusoidal forcing function, respectively.

In technical terms, this system is categorized as a non-smooth Filippov system with a degree of smoothness (DoS) of $1[9,18]$. Due to the Coulomb friction, the dynamics of this system become discontinuous at plane $\Sigma=\dot{u}-1=0$, famously called the switching manifold, where the solution trajectories are attracted from above and below to form a sliding region $\hat{S}$ (region with attracting sliding motion in the close neighbourhood of $\Sigma$ ). For the investigation of dynamical scenarios if we let the amplitude $F=0.1$ [27], but allow the frequency $\varpi$ to be varied as a bifurcation parameter, the sliding motion leads to an interesting bifurcation close to $\varpi \simeq 1.7078$ where the sliding orbit suddenly loses its stability to a chaotic orbit (see Figs. 


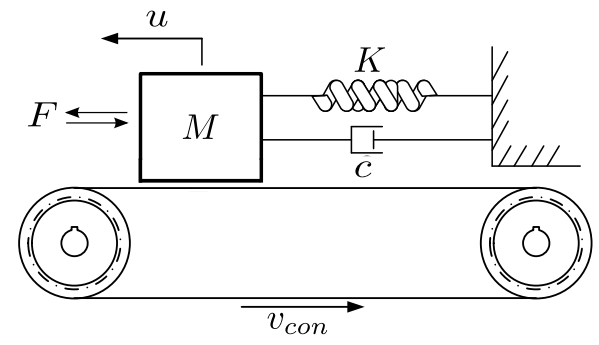

Fig. 1. Model of a forced oscillator with dry friction.
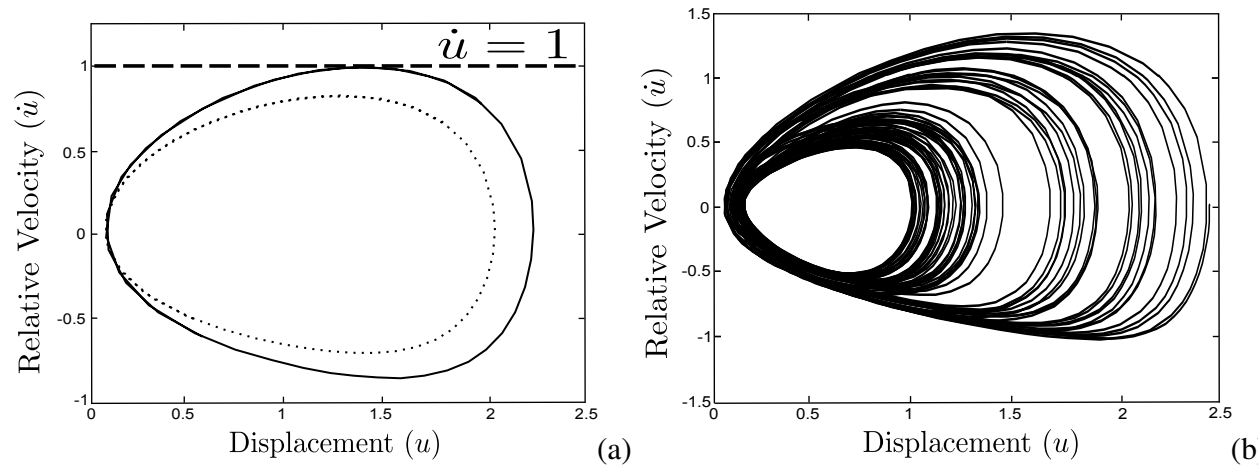

Fig. 2. (a) $4 T(8 \pi / \bar{\sigma})$ periodic orbit grazes the boundary of sliding region (grazing-sliding event) at $\Phi=1.7077997$ and turns to a (b) chaotic orbit. Dot-dashed line in (a) shows a stable, non-sliding periodic orbit for $\bar{\omega}=1.7082$ before sliding-grazing event, and the solid line in (a) shows the sliding orbit near the tangency of attracting switching manifold.

2 and 3$)$.

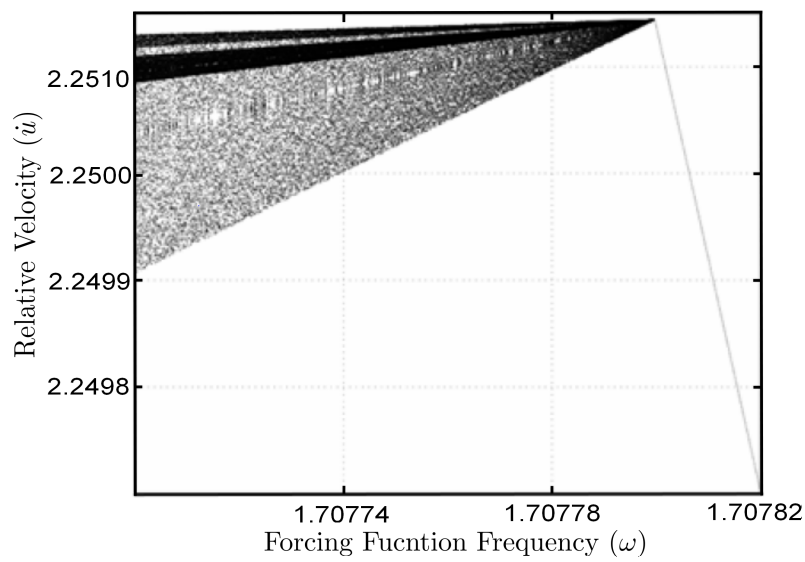

Fig. 3. The bifurcation diagram shows the grazing-sliding bifurcation where there is a sudden transition to a chaotic attractor.

The sliding-grazing bifurcation occurs as the sliding $4 T$-periodic orbit (the orbit depicted with solid line in Fig. 2a) becomes tangential (grazing) to the switching manifold, where the boundary of the sliding region becomes the switching manifold $\dot{u}-1=0$ itself. This scenario arises when the dry-friction oscillator tries to immediately enter a stick-slip phase from a purely slip phase so the attracting orbit is placed precisely at the transition (bifurcation) point. When the transition occurs, the orbit abruptly becomes chaotic as seen in Fig. 2 b (see also Fig. 4 for the illustration of sliding-grazing scenario). 


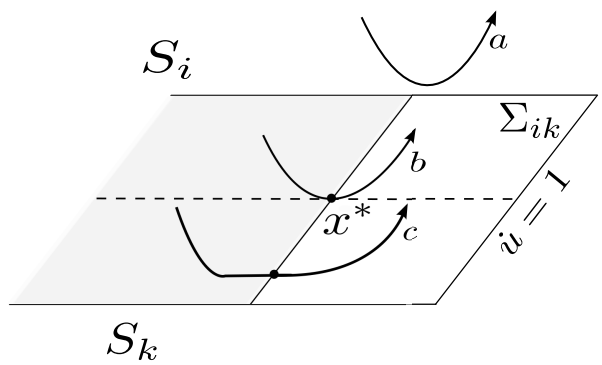

Fig. 4. With small parameter variation, periodic (sliding) orbit $a \rightarrow b$, touches the switching manifold $\Sigma$ at $x^{*}$ undergoing a sliding-grazing bifurcation, and $b \rightarrow c$. In fact, periodic orbits $a$ and $c$ might exist for the same parameters values.

\subsubsection{Non-smooth TS fuzzy model}

There is a rich literature on fuzzy model-based control using Takagi-Sugeno (TS) fuzzy modelling [22-26] for representing non-linear, smooth dynamical systems. This modelling approach is mainly developed to provide a multi-parametric stability analysis framework as it essentially divides the whole state space into a number of local smooth dynamics where the interpolations of the linear local dynamics are describe by a set of fuzzy implications [22]. However, as the authors have discussed in previous publications $[17,21]$ this model cannot describe the discontinuous property of non-smooth Filippov systems (and mechanical impacting systems) and all the arising DIBs. In this section, a non-smooth TS fuzzy formalism is proposed for Filippov systems able to describe the sliding dynamics local to the discontinuity boundary.

To describe such formalism, first we should describe the basic smooth TS fuzzy model ${ }^{2}$ as follows:

$$
\dot{x}(t)=\sum_{j=1}^{l} \mu^{j}(x(t))\left(A^{j} x(t)+B^{j} u(t)\right)
$$

where $x(t) \in \mathfrak{R}^{n}$ is the state vector, $u(t) \in \mathfrak{R}^{m}$ is the control input vector, $A^{j} \in \mathfrak{R}^{n \times n}$ and $B^{j} \in \mathfrak{R}^{n \times m}$ are respectively the system matrix and the input matrix. $\mu^{j}(x(t))$ is the degree of membership described by $\mu^{j}(x(t))=\omega^{j}(x(t)) / \sum_{k=1}^{l} \omega^{k}(x(t))$, where $\omega^{j}(x(t)) \geq 0, \sum_{k=1}^{l} \omega^{k}(x(t))>0, k=1,2, \ldots, l$ ( $l$ is the number of model rules). The weighting function can be described by $\omega^{j}(x(t))=\prod_{i=1}^{n} \Gamma_{i}^{j}\left(x_{i}(t)\right)$ derived by a series of model rules as:

Model Rule $j: \operatorname{IF} x_{1}(t)$ is $\Gamma_{1}^{j}$ AND...AND $x_{n}(t)$ is $\Gamma_{n}^{j}$ THEN $\dot{x}=A^{j} x(t)+B^{j} u(t), j=1,2, \ldots, l$,

where $\Gamma_{i}^{j}$ is a fuzzy set.

To represent a sliding Filippov systems (DoS of one), we propose to extend (5) to a non-smooth TS fuzzy model as

\footnotetext{
${ }^{2}$ We mean smooth TS fuzzy model as the typical TS fuzzy model able to approximate smooth nonlinear functions.
} 
described by the following model rules:

$$
\text { Model Rule } j: \text { IF } x_{1} \text { is } \Gamma_{1}^{j} \text { AND } \ldots \text { AND } x_{n} \text { is } \Gamma_{n}^{j} \text { THEN }\left\{\begin{array}{l}
\dot{x}=\sum_{j=1}^{l_{m}} \mu^{j}(x, m)\left(A^{j}(m) x+B^{j}(m) u\right) \\
m^{+}=\xi(x, m),
\end{array}\right.
$$

where the embedded discrete function $m^{+}(t)=\xi(x(t), m(t))$ describes the switching (or discrete) events between continuous states $x \in R^{n}$ on or around the switching manifold $\Sigma$, and $m \in M=\left\{m_{1}, \ldots, m_{N}\right\}$ is a discrete state variable associated with a local continuous dynamic $f\left(x, m_{i}\right)$ where the discontinuous change to another $f\left(x, m_{j}\right)$ can only take place if we let the change to the next discrete state $\xi: m_{i} \mapsto m_{j}$ (notation $m^{+}$means the next state of $m$ ). Therefore, the local smooth dynamics associated with discrete state $m_{i}$ is represented by a specific set, which we will call a fuzzy sub-vector field, denoted by $F_{m_{i}}$ and described as:

$$
F_{m_{i}}=\sum_{j \in\{1,2, \ldots\}} \mu^{j}\left(x, m_{i}\right)\left(A^{j}\left(m_{i}\right) x+B^{j}\left(m_{i}\right) u\right)
$$

where $A^{j}\left(m_{i}\right) \in \mathfrak{R}^{n \times n}, B^{j}\left(m_{i}\right) \in \mathfrak{R}^{n \times m}$ and $\mu^{j}: \mathfrak{R}^{n} \times M \rightarrow[01], i \in I_{N}=\{1,2, \ldots, N\}$, are continuous weighting functions satisfying $\sum_{j=1}^{l_{m}} \mu^{j}(x, m)=1$ and $l_{m}$ is the number of fuzzy rules.

Remark 1: In order to describe rapid switching of sliding dynamics in (6), the function $\xi$ is substituted by a number of switch sets as

$$
S_{i, k}=\left\{x \in \mathfrak{R}^{n} \mid m_{k}=\xi\left(x, m_{i}\right)\right\}, i, k \in\{1,2, \ldots, N\},
$$

The switch sets simply describe where in the continuous state space a fuzzy sub-vector field $F_{m_{i}}$ switches to another $F_{m_{k}}$ provided that $m_{i} \neq m_{k}$

In the model (6), sliding dynamics can be described if two switch sets $S_{i, k}$ and $S_{k, i}$ coincide and form a sliding manifold which, in our case, resides on switching manifold $\Sigma$ itself. This means that the two fuzzy sub-vector fields $F_{m_{k}}$ and $F_{m_{i}}$ approach very close to $\Sigma$ and slide along it. However, this scenario results in an uncertainty of infinite number of rapid switches between the discrete states $m_{i}$ and $m_{k}$ where it is very difficult to ascertain the value of discrete states at any time in the sliding mode. To resolve such an uncertainty problem, a new discrete state like $m_{z}$ is introduced in $\xi(x, m)$ to define the sliding dynamics as the infinite number of switching between $m_{i}$ and $m_{k}$ occurs. In this case, the switching (sliding) manifold can be readily represented by switch sets $S_{i, z}$ and $S_{i, k}$. More importantly, the existence and uniqueness of solution near the sliding manifold can be held. As a result, the sliding dynamics along the switching manifold can be reformulated in the model (6) using the classical methods of Filippov's convex combination or Utkin's equivalent control [28]. 
A fuzzy sub-vector field $F_{m}(7)$ basically represents smooth dynamics in the same way as model (4) approximates a smooth dynamical system. Hence, we can adopt a linearisation transformation approach [23] for the approximation of continuous part of dynamics in a non-smooth system of the form (6). However, we replace the operating regions of fuzzy sub-systems in (7) with flexible regions defined as $\Omega_{q}^{x, m_{i}, j} \subset \mathcal{F}$ ( $\mathcal{F}$ denotes the whole state space comprised of discrete and continuous fuzzy states) to represent $F_{m_{i}}$ in $\triangle$ flexible regions. In this way, the regions can be defined as a support set of continuous fuzzy states:

$$
[\mu]^{0}=\left\{x \in \Omega_{q}^{x, m_{i}} \mid \mu^{j}\left(x, m_{i}\right)>0\right\}
$$

where each region $\Omega_{q}^{x, m_{i}, j}$ is designated to $[\mu]^{0}$ and defines to what degree the local terms $A^{j}\left(m_{i}\right) x+B^{j}\left(m_{i}\right) u$ are a part of $F_{m_{i}}$ and hence, the overall dynamics. With $[\mu]^{0}$, a non-smooth model (6) is obtained by letting the fuzzy variables $z_{i}(x)$ to be defined as a convex combination of flexible region boundaries

$$
z_{i}(x)=M\left(z_{i}(x)\right) \cdot \min _{x}[w]^{0}+\left(1-M\left(z_{i}(x)\right)\right) \cdot \max _{x}[w]^{0}
$$

where $\min _{x}[w]^{0}$ and $\max _{x}[w]^{0}$ are respectively the lower boundary and the upper boundary of flexible regions $\Omega_{q}^{x, m_{i}, j}$. It will be shown in the next section that the existence of such flexible regions is essential for the structural stability analysis.

To construct a non-smooth model of the oscillator motion (2) with the nonlinear Coulomb friction law (3) based on the formalism (6), (7) and (8), we define $x_{1}=u$ and $x_{2}=\dot{u}$, and two fuzzy set supports $[w 1]^{0}$ and $[w 2]^{0}$ for the state variable $x_{2}$, with the respective boundaries of $x_{2} \in[-0.5,1]$ and $x_{2} \in[1.001,2.5]$. We also define two fuzzy variable $z_{1}$ and $z_{2}$ for the nonlinear term $\alpha_{1}+2 \alpha_{2}+\alpha_{2}\left(x_{2}^{2}+2 x_{2}\right)$, respectively, based on $[w 1]^{0}$ and $[w 2]^{0}$. Using the definition (10), the fuzzy variables are calculated as $z_{1}(t)=M_{1}\left(z_{1}(t)\right) \times 2.9625+M_{2}\left(z_{1}(t)\right) \times 3.7500$, and $z_{2}(t)=M_{3}\left(z_{2}(t)\right) \times 3.7518+M_{4}\left(z_{2}(t)\right) \times 7.4625$ where $M_{1}, M_{2}, M_{3}$ and $M_{4}$ are fuzzy sets or membership functions, which are obtained as

$$
\begin{array}{ll}
M_{1}\left(z_{1}(t)\right)=\left(z_{1}(t)-3.7500\right) / 0.7875, & M_{2}\left(z_{1}(t)\right)=\left(2.9625-z_{1}(t)\right) / 0.7875 \\
M_{3}\left(z_{2}(t)\right)=\left(z_{2}(t)-7.4625\right) / 3.7107, & M_{4}\left(z_{2}(t)\right)=\left(3.7518-z_{2}(t)\right) / 3.7107 .
\end{array}
$$

and $\xi(x, m)$ is described by switch sets $(8)$ :

$$
S_{1,2}=\left\{x \in R^{n} \mid 1-x_{2}(t)>0\right\}, \quad S_{2,1}=\left\{x \in R^{n} \mid 1-x_{2}(t)<0\right\},
$$


which explains the interaction between the fuzzy sub-vector fields $F_{m_{1}}$ and $F_{m_{2}}$, where they are composed of the following sub-systems respectively:

$$
\begin{aligned}
A^{1}\left(m_{1}\right)= & {\left[\begin{array}{cc}
0 & 1 \\
-1 & 2.9625
\end{array}\right], A^{2}\left(m_{1}\right)=\left[\begin{array}{cc}
0 & 1 \\
-1 & 3.7500
\end{array}\right], A^{3}\left(m_{1}\right)=\left[\begin{array}{cc}
0 & 1 \\
-1 & 3.7518
\end{array}\right], A^{4}\left(m_{1}\right)=\left[\begin{array}{cc}
0 & 1 \\
-1 & 7.4625
\end{array}\right], } \\
B^{j}\left(m_{1}\right)= & {\left[\begin{array}{c}
0 \\
\alpha_{0}-\alpha_{1}+\alpha_{2}+F \cos (\varpi t)
\end{array}\right], j=1,2,3,4, }
\end{aligned}
$$

and

$$
\begin{aligned}
A^{1}\left(m_{2}\right) & =\left[\begin{array}{cc}
0 & 1 \\
-1 & 2.9625
\end{array}\right], A^{2}\left(m_{2}\right)=\left[\begin{array}{cc}
0 & 1 \\
-1 & 3.7500
\end{array}\right], A^{3}\left(m_{2}\right)=\left[\begin{array}{cc}
0 & 1 \\
-1 & 3.7518
\end{array}\right], A^{4}\left(m_{2}\right)=\left[\begin{array}{cc}
0 & 1 \\
-1 & 7.4625
\end{array}\right], \\
B^{j}\left(m_{2}\right) & =\left[\begin{array}{c}
0 \\
-\alpha_{0}-\alpha_{1}+\alpha_{2}+F \cos (\varpi t)
\end{array}\right], j=1,2,3,4 .
\end{aligned}
$$

Hence, the model rules for dry-friction oscillator are expressed as follows:

$$
\text { Model Rule j: IF } x_{2}(t) \text { is } \Gamma^{j} \operatorname{THEN}\left\{\begin{array}{l}
\dot{x}(t)=A^{j}\left(m_{i}\right) x(t)+B^{j}\left(m_{i}\right) u(t), j=1,2,3,4, i=1,2, \\
m^{+}=\xi(x, m),
\end{array}\right.
$$

To better understand the model above and the interaction of fuzzy sub-vector fields when trajectories graze and slide near the switching manifold, we describe the TS fuzzy model (12) in the form of differential inclusions as follows:

$$
\dot{x}=f=\left\{\begin{array}{l}
\left\{F_{m_{1}}=\sum_{j}^{4} \mu^{j}\left(x, m_{1}\right)\left(A^{j}\left(m_{1}\right) x+B^{j}\left(m_{1}\right) u\right)\right\}, \text { if } x \in S_{1,2}, \\
\left\{F_{m_{2}}=\sum_{j}^{4} \mu^{j}\left(x, m_{2}\right)\left(A^{j}\left(m_{2}\right) x+B^{j}\left(m_{2}\right) u\right)\right\}, \text { if } x \in S_{2,1}, \\
\left\{\mu F_{m_{1}}+(1-\mu) F_{m_{2}} \mid 0<\mu<1\right\}, \text { if } x \in \partial S_{1} \cap \partial S_{2} .
\end{array}\right.
$$

The third term of $f$ means that the interaction of the fuzzy sub-vector fields $F_{m_{1}}$ and $F_{m_{2}}$ (based on (11)) near the switching manifold $\left(x \in \partial S_{1} \cap \partial S_{2}\right)$ is described as the convex combination of $F_{m_{1}}$ and $F_{m_{2}}$, which naturally holds for TS fuzzy models as a poly-topic system. Furthermore, the third term of $f$ describes the familiar equivalent sliding dynamics according to 


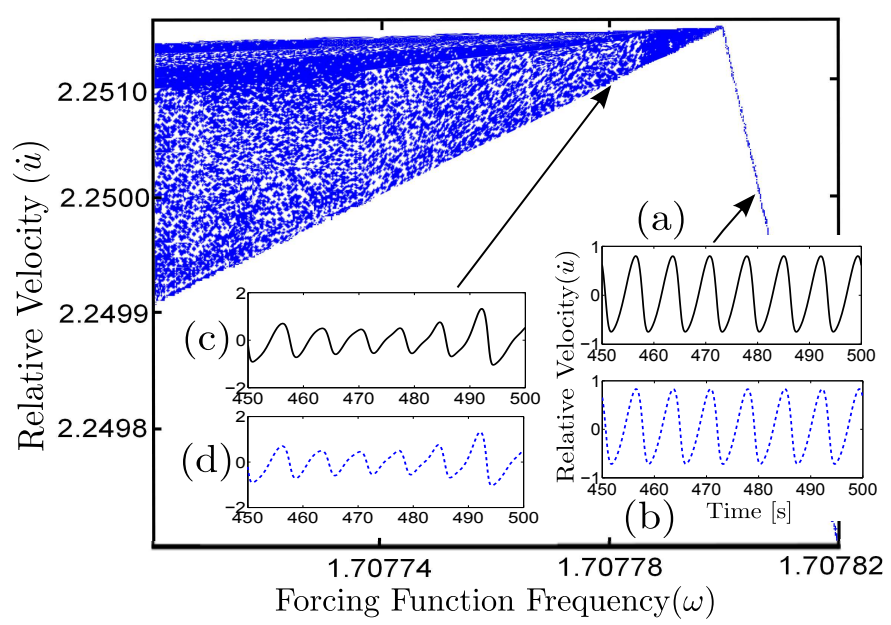

Fig. 5. Bifurcation diagram obtained from the direct simulation of the non-smooth TS fuzzy model (11),(12) shows the grazing-sliding DIB at $\bar{\varpi}=1.70778$. Small figures show steady-state time responses.

Filippov [1] close to switching manifold. The equivalent dynamics can be alternatively formulated by Utkin's method as explained immediately below.

Fuzzy sub-vector fields $F_{m_{1}}$ and $F_{m_{2}}$ represent the solution trajectories with opposite directions in the close vicinity of the switch sets (11); hence, coinciding the two switch sets forms an attractive sliding region $\hat{S}$. As mentioned, by introducing a new discrete state $m_{z}$, the sliding dynamics can be described with Utkin's equivalent control if we assume that the manifold $S=\left\{x \in R^{n} \mid 1-x_{2}(t)=0\right\}$ is formed by coinciding $S_{1,2}$ and $S_{2,1}$ as $F_{m_{1}, m_{2}}=\left\{\left(F_{m_{1}}+F_{m_{2}}\right) / 2\right\}+\left\{\left(F_{m_{1}}+F_{m_{2}}\right) / 2\right\} U_{e q}$ where $F_{m_{1}, m_{2}}=F_{m_{z}}$ denotes a sliding fuzzy sub-vector field and the equivalent sliding dynamics $U_{e q}$ is formulated as:

$$
U_{e q}=-\frac{\nabla S \cdot F_{m_{1}}+\nabla S \cdot F_{m_{2}}}{\nabla S \cdot F_{m_{2}}-\nabla S \cdot F_{m_{1}}}=-\frac{F_{m_{1}}+F_{m_{2}}}{F_{m_{2}}-F_{m_{1}}},
$$

where $U_{e q} \in[-1,1], \nabla S=\partial S / \partial x$ and the sliding region $\hat{S}$ is defined as $\hat{S}=\left\{x \in S:-1 \leq U_{e q} \leq 1\right\}$.

Comparing the bifurcation diagrams of the non-smooth TS fuzzy model (11), (12) with that of a physical model of the forced oscillator (see Figs. 5 and 3) validates the ability of the new formalism to model the non-smooth sliding dynamics and the resulting sliding-grazing DIB. The accuracy of modelling is also visible in the steady-state time responses of the stable periodic orbit at forcing function frequency $\varpi=1.70781$ (see small Figs. 5a and 5b where the solid lines represents the oscillator response and blue-coloured dotted-line represents TS fuzzy model) and when the periodic orbit undergoes a DIB at $\varpi=1.70778$ and becomes chaotic (see small Figs. 5c and 5d).

\section{Structural stability analysis}

A Lyapunov framework is proposed in this section for the analysis of the sliding-grazing bifurcation in a dry-friction oscillator. The proposed approach is based on the constructed TS fuzzy model (12), and the employment of non-smooth Lyapunov functions where the energy functions are piecewise continuous in $\triangle$ detached but flexible regions of fuzzy state 
space $\mathcal{F}$. This is an essential step to deal with the complex scenarios of the sliding-grazing periodic orbit with respect to the switching manifold and to avoid the conservative formulation when recasting the stability theorems into Linear Matrix Inequalities (LMI) conditions. If we let a region $\Omega$ be defined as a support set as described in (9), then we can define the subsets $\Omega^{x}=\left\{x \in \mathfrak{R}^{2} \mid(x, m) \in \Omega\right\}$ including continuous fuzzy states, $\Omega^{x, m_{i}}=\left\{x \in \mathfrak{R}^{2} \mid(x, m) \in \Omega\right\}$ including continuous fuzzy states and $\Omega^{m}=\{m \in M \mid(x, m) \in \Omega\}$ including discrete fuzzy states. We further let $\Omega \subseteq \mathcal{F}$ be partitioned such that $\Omega_{1} \cup \ldots \cup \Omega_{\Delta}=\Omega, \Omega_{q} \cap \Omega_{r}=\emptyset, q \neq r, q, r \in I_{\Delta}$ where $I_{\Delta}$ is a set containing the number of $\Delta$ detached regions. For hyperplanes, or boundaries of a region, we define region $\Lambda_{q r}, q, r \in I_{\Delta}, q \neq r$ as a set of fuzzy states for which the solution trajectory satisfies (11), (12), with initial states $\left(x_{0}, m_{0}\right) \in \mathcal{F}_{0}$, and passes from $\Omega_{q}$ to $\Omega_{r}$, i.e. $\Lambda_{q r}=\{(x, m) \in \Omega \mid \exists t<$ $t_{0}$, such that $\left.\left(x\left(t^{-}\right), m\left(t^{-}\right)\right) \in \Omega_{q},(x(t), m(t)) \in \Omega_{r}\right\}$. In order to allow the solution trajectories to move through from one region onto another we define a set of tuples $\mathrm{I}_{\Lambda}=\left\{(q, r) \mid \Lambda_{q r} \neq \emptyset\right\}$ which indicates there is at least one point for which the solution trajectory can move through from $\Omega_{q}$ to $\Omega_{r}$. Considering the region partitions, the non-smooth Lyapunov function candidate is defined as:

$$
V(x)=V_{q}(x) \text { when }(x, m) \in \Omega_{q}
$$

where $V(x)$ is a non-smooth Lyapunov function at the boundary regions $\Lambda_{q r},(q, r) \in I_{\Lambda}$ and $V_{q}: \operatorname{cl} \Omega_{q}^{x} \rightarrow \Re, q \in I_{\Delta}$ representing the system's (abstract) energy in each local region $\Omega_{q}$ (cl. denotes the closure of a set, which is the smallest closed set containing the set). $V(x)$ is also considered piecewise continuous as a function of time. To formulate the stability problem as LMI, $V_{q}(x)$ in (13) is defined as piecewise quadratic matrices $V_{q}(x)=\pi_{q}+2 p_{q}^{T} x+x^{T} P_{q} x,(x, m) \in \Omega_{q}, q \in I_{\Delta}, \pi_{q} \in \mathfrak{R}$, $p_{q} \in \mathfrak{R}^{n}$ and $P_{q}=P_{q}^{T} \in \mathfrak{R}^{n} \times \mathfrak{R}^{n}$. For ease of formulation we can rewrite $V_{q}(x)=\tilde{x}^{T} \tilde{P}_{q} \tilde{x}$ where the matrices $\tilde{x}=\left[\begin{array}{ll}x & 1\end{array}\right]^{T}$ and $\tilde{P}_{q}=\left[\begin{array}{lll}P_{q} & p_{q} ; p_{q}^{T} \pi_{q}\end{array}\right]$. Since all the stability conditions presented in the subsequent theorem 1 must be confined to local regions $\Omega_{q}^{x}, \Omega_{q}^{x, m_{i}}, \Omega_{q}^{m}$ and the boundary regions $\Lambda_{q, r}^{x}$, the regions are formulated as quadratic inequalities recastable to LMI conditions. This is possible if the confined regions are defined as quadratic functions and then transformed to unconfined conditions using the well-known $S$-procedure technique [29]. Therefore, if we define a region as a confined inequality condition $Q^{0}(x) \geq 0, \forall x \in \Omega$ where $Q^{0}(x): \Re^{n} \rightarrow \Re$ is a function with unknown variables to be determined, the condition can be substituted with an unconfined condition $Q^{0}(x) \geq \sum_{k=1}^{s} \lambda^{k} Q^{k}(x), \forall x \in \Re^{n}, \exists \lambda^{k} \geq 0, k \in I_{s}$ (see Lemma in [29]). Then by defining

$Q^{k}(x)=x^{T} Z^{k} x+2\left(c^{k}\right)^{T} x+d^{k}=\tilde{x}^{T} \tilde{Z}^{k} \tilde{x},(k=0, \ldots, s), \tilde{x}=\left[\begin{array}{l}x \\ 1\end{array}\right], \tilde{Z}^{k}=\left[\begin{array}{cc}Z^{k} & c^{k} \\ \left(c^{k}\right)^{T} & d^{k}\end{array}\right], Z^{k}=\left(Z^{k}\right)^{T} \in \Re^{n} \times \Re^{n}, c^{k} \in \mathfrak{R}^{n}, d^{k} \in \mathfrak{R}$

we transform the unconfined condition to LMI as $\tilde{Z}^{0} \geq \sum_{k=1}^{s} \lambda^{k} \tilde{Z}^{k}, \quad \lambda^{k} \geq 0, k \in I_{s}$.

Note: Boundary regions $\Lambda_{q r}$ can represent the switching manifolds (or switch sets in (11)). This type of regions can 
be substituted by quadratic forms $Q^{k}(x)=0, k \in I_{s}$, making the imposition of constraints on additional parameters such as $\lambda^{k} \geq 0, k \in I_{s}$ unnecessary since the inequality $Q^{0}(x) \geq \sum_{k=1}^{s} \lambda^{k} Q^{k}(x)$ is true regardless of the sign of these parameters. In this case, matrices $\tilde{Z}^{k}$ replacing the regions $\Lambda_{q r}$ can be denoted as $\tilde{Z}_{q, r}^{k}$.

With having a method for LMI formulation of regions, the fundamental theorem for the structural stability analysis can be presented as a LMI stabilization problem as follows:

Theorem 1. If there exist piecewise quadratic matrices $\tilde{P}_{q}, q \in I_{\Delta}$, constants $\eta_{k}^{q r}$ and if there is a solution to min $\beta$ subject to

1. $\alpha>0, \mu_{q}^{k} \geq 0, \hat{\mu}_{q}^{k} \geq 0, v_{q i j}^{k}>0$,

2. $\left[\begin{array}{ll}\alpha & 0 \\ 0 & 0\end{array}\right]+\sum_{k=1}^{s_{q}} \mu_{q}^{k}\left[\begin{array}{cc}Z_{q}^{k} & c_{q}^{k} \\ \left(c_{q}^{k}\right)^{T} & d_{q}^{k}\end{array}\right] \leq \tilde{P}_{q}$,

3. $\tilde{P}_{q} \leq\left[\begin{array}{ll}\beta & 0 \\ 0 & 0\end{array}\right]+\sum_{k=1}^{s_{q}} \hat{\mu}_{q}^{k}\left[\begin{array}{cc}Z_{q}^{k} & c_{q}^{k} \\ \left(c_{q}^{k}\right)^{T} & d_{q}^{k}\end{array}\right], q \in I_{\Delta}$,

4. $\left[\begin{array}{ll}\left(A^{j}\left(m_{i}\right)\right)^{T} P_{q}+P_{q} A^{j}\left(m_{i}\right) & P_{q} B^{j}(m)+\left(A^{j}\left(m_{i}\right)\right)^{T} p_{q} \\ \left(B^{j}\left(m_{i}\right)\right)^{T} P_{q}+p_{q}^{T} A^{j}\left(m_{i}\right) & \left(B^{j}\left(m_{i}\right)\right)^{T} p_{q}+p_{q}^{T} B^{j}\left(m_{i}\right)\end{array}\right]+\sum_{k=1}^{s_{q i j}} v_{q i j}^{k}\left[\begin{array}{cc}Z_{q}^{k} & c_{q}^{k} \\ \left(c_{q}^{k}\right)^{T} & d_{q}^{k}\end{array}\right] \leq-\tilde{I}, q \in I_{\Delta}, m_{i} \in \Omega_{q}^{m}$,

5. $\tilde{P}_{r} \leq \tilde{P}_{q}-\sum_{k=1}^{s_{q r}} \eta_{k}^{q r}\left[\begin{array}{cc}Z_{q r}^{k} & c_{q r}^{k} \\ \left(c_{q r}^{k}\right)^{T} & d_{q r}^{k}\end{array}\right],(q, r) \in I_{\Lambda}$,

then the invariant set is structurally stable in the sense of Lyapunov (i.e. the periodic solution exponentially converges to the stable fixed point of the Poincaré map). ${ }^{3}$

Remark 2: Theorem 1 links the idea of asymptotic (exponential) Lyapunov stability with the novel notion of structural stability. As we deal with the stability of more complex scenarios of periodic orbits, we assume that the system states (x,m) must converge to the stable fixed point, the intersection of the stable periodic orbit of interest with the Poincare map [9]. In this way, the structural stability, can be verified by the exponential (asymptotic) stability of the fixed point in the sense of Lyapunov

Remark 3: To derive the rate of exponential convergence, the conditions are first scaled by $1 / \gamma$ and then the LMI optimization problem can be solved when $\beta$ is minimized. If we search for the maximal value of $\alpha$ when trying to minimize $\beta$, the computational effort will be minimized. The maximal value of $\alpha$ is the smallest eigenvalue of all matrices $P_{q}$, should all regions contain the origin as an interior point. This is mainly due to $\lambda_{q}^{\min } x^{T} x \leq x^{T} P_{q} x$ [30] where $\lambda_{q}^{\min }$ is the smallest eigenvalue of $P_{q}$

In theorem 1, the search for $V(x)$ in (13) is formulated in flexible regions (except for boundary regions $\Lambda_{q r}$ representing switch sets). Determining the size of the regions $\Omega_{q}$ is important since if a region are substituted by quadratic inequalities covering too large a region, the LMI stabilization problem will be found infeasible due to the conservative formulation. As

\footnotetext{
${ }^{3}$ The essential part of the proof is similar to the proof presented for non-sliding Filippov systems [21] (DoS of 1). The extended detail of the proof for sliding dynamics including the existence and uniqueness of the solution when there is a rapid switching of sliding motion near the switching manifold is omitted here due to insufficient space.
} 
will be shown later finer region partitions are needed in the close neighbourhood of the switching manifold to verify structural stability. However, the sliding-grazing event occurs in such a narrow domain of parameter variation, that the use of trial and error approach for the selection of size and number of partitions would be, at best, an exhaustive process. An extension to theorem 1 is needed to automate the region partitioning process by finding finer regions close to the sliding region.

As pointed out, in substituting the regions $\Omega_{q}$ with quadratic inequalities, the inequality $Q^{k}(x) \geq 0$ shall not cover a larger region than $\Omega_{q}$. Even though the whole state space $\Omega \subseteq \mathcal{F}$ is naturally partitioned by switch sets $S_{i, j}$ (then replaced by $\Lambda_{q r}$ ) according to the definition of $V(x)$ in (13), this partitioning will not suffice the feasibility conditions since in the neighbourhood of sliding-grazing orbit, boundary regions $\Lambda_{q r}$ take an arbitrary form. Therefore, before the substitution by the terms $Q^{k}(x) \geq 0$, region $\Omega$ must be restricted by more hyperplanes to achieve arbitrary region partitioning with more exact coverage. To describe the automation procedure, first, we have to describe how the hyperplanes, i.e. the boundary region $\Lambda_{q r}$, is represented by quadratic inequalities. If $\Lambda_{q r}$ is substituted by a set of states fulfilling quadratic equalities of the form $f^{T} x+g=0, f=\left[f^{1} \ldots f^{n}\right]^{T} \in \mathfrak{R}^{n}, g \in \mathfrak{R}$, the quadratic form becomes equivalent to the quadratic equalities

$$
\tilde{x}^{T}\left[\begin{array}{c}
\lambda \\
\lambda^{n+1}
\end{array}\right]\left[f^{T} g\right] \tilde{x}+\tilde{x}^{T}\left[\begin{array}{l}
f \\
g
\end{array}\right]\left[\lambda^{T} \lambda^{n+1}\right] \tilde{x}=\sum_{k=1}^{n+1} \lambda^{k} \tilde{x}^{T} \tilde{Z}^{k} \tilde{x}=0
$$

where $\tilde{Z}^{k}=e^{k}\left[f^{T} g\right]+\left[f^{T} g\right]^{T}\left(e^{k}\right)^{T}, \tilde{x}=\left[\begin{array}{ll}x & 1\end{array}\right]^{T}, \lambda=\left[\lambda^{1}, \ldots, \lambda^{n}\right]^{T} \in \mathfrak{R}^{n}, \lambda^{n+1} \in \mathfrak{R}$ are arbitrary additional parameters and $e^{k}$ is a column vector with $n$ elements such that $e^{k}(i)=1, i=k$ and $e^{k}(i)=0, i \neq k\left(i\right.$ means the $i^{t h}$ element of $\left.e^{k}\right)$. The search for arbitrary region partitions is presented in the following routine:

Routine 1. If there exist piecewise quadratic matrices $\tilde{P}_{q}, q \in I_{\Delta}$, constants $\eta_{k}^{q r}$, and if there is a solution to min $\beta$ subject to the following routine

1. $\xi_{0}=1, a=0, \delta_{0}=0$,

2. $g_{a} \leq g_{0}+\delta_{a}, g_{0}+\delta_{a} \leq g_{a}$,

3. $2\left(\lambda^{T} x+\lambda^{n+1}\right)^{T}\left(f^{T}+g_{a}\right)=0$,

4. Stability conditions 1, 2, 3, 4 \& 5 of Theorem 1,

5. If not feasible cont inue otherwise stop

6. $a=a+1, \delta_{a}=\delta_{a}+c \cdot \xi_{a}, \xi_{a}=\xi_{a} \cdot \varepsilon$,

7. Goto 2

then the sliding-grazing invariant set is structurally stable in the sense of Lyapunov.

As previously mentioned, the switching manifold $\Sigma=\left\{x \in \mathfrak{R}^{n} \mid 1-x_{2}(t)=0\right\}$ is an intrinsic hyper-plane partitioning the fuzzy state space $\mathcal{F}$ to regions $\Omega_{1}$ and $\Omega_{2}$ where on the boundary $\Lambda_{12}$ the switch sets $S_{1,2}$ and $S_{2,1}$ coincides and form the sliding region $\hat{S}$. Therefore, we initiate Routine 1 with the selection $f=[0-1]$ and $g=1$. We can set the value for $c$ and initial value for $\xi_{a}$ as any value; nonetheless, as the distribution of partitions need to be formed closer to $\hat{S}$, we set 
the initial values $c=0.1$ and $\xi_{a}=0.01$ to achieve the result with less computational effort. Varying the forcing function frequency $\Phi$ as the bifurcation parameter while running Routine 1 results in a feasible solution exactly at the bifurcation point of $\Phi=1.70779$ (see Fig. 2 and 5), with the following piecewise quadratic matrices and 8 region partitions (see the region distribution in Fig. 6):

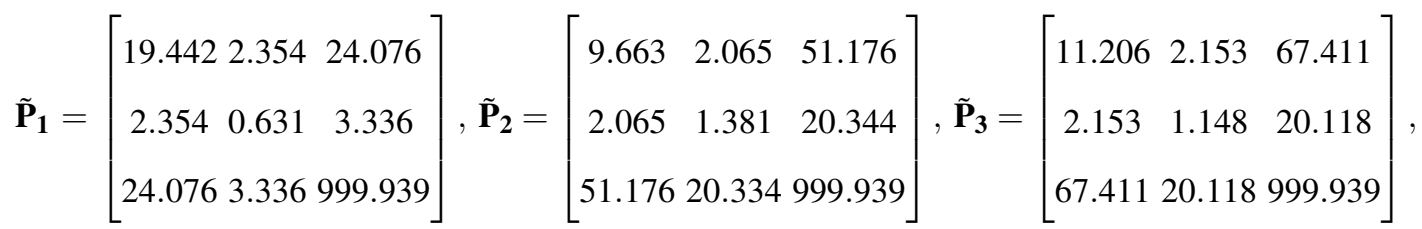

$$
\begin{aligned}
& \tilde{\mathbf{P}}_{\mathbf{4}}=\left[\begin{array}{ccc}
11.209 & 2.153 & 67.448 \\
2.153 & 1.148 & 20.117 \\
67.448 & 20.117 & 999.939
\end{array}\right], \tilde{\mathbf{P}}_{\mathbf{5}}=\left[\begin{array}{ccc}
9.663 & 2.065 & 51.176 \\
2.065 & 1.381 & 20.344 \\
51.176 & 20.344 & 999.851
\end{array}\right], \tilde{\mathbf{P}}_{\mathbf{6}}=\left[\begin{array}{ccc}
11.206 & 2.153 & 67.411 \\
2.153 & 1.148 & 20.118 \\
67.411 & 20.118 & 999.939
\end{array}\right], \\
& \tilde{\mathbf{P}}_{7}=\left[\begin{array}{ccc}
11.209 & 2.153 & 67.448 \\
2.153 & 1.148 & 20.117 \\
67.448 & 20.117 & 999.851
\end{array}\right], \tilde{\mathbf{P}}_{\mathbf{8}}=\left[\begin{array}{ccc}
19.440 & 2.354 & 141.477 \\
2.354 & 0.628 & 19.601 \\
141.477 & 19.601 & 999.939
\end{array}\right]
\end{aligned}
$$

The exponential convergence to the fixed point is optimized with the value of $\beta=36.295$. This means that the sliding orbit is exponentially stable in the sense of Lyapunov (see Remark 2). For any frequency less than $\Phi=1.70779$, Routine 1 would result in infeasibility of the solution, which is the analytical confirmation of the instability of the $4 T$ periodic orbit which grazes the sliding region and undergoes the sliding-grazing bifurcation (see Fig. 2). Thus the onset of the sudden chaotic attractor can be analytically predicted using Routine 1 (along with Theorem 1). As can be noticed in Fig. 6, the hyperplanes resulting from Routine 1 introduce progressively narrower regions close to the first boundary region

$$
\Lambda_{q r}=2\left(\lambda^{T} x+\lambda^{n+1}\right)^{T}\left([0-1]^{T} x+1\right)=0,
$$

where the parameters $\lambda$ is determined by solving the quadratic inequalities as described.

\section{Conclusions}

The stability of the complex sliding-grazing scenario in a forced dry-friction oscillator is investigated in this paper. A TS fuzzy model-based approach is suggested to analytically predict the onset of such a unique non-smooth bifurcation leading to an abrupt chaotic orbit. It is shown that using the proposed non-smooth TS fuzzy formalism, the sliding dynamics formed by discontinuous switching can be modelled to exhibit all the observed non-linearities in the physical model. Structural stability of a local grazing-sliding orbit is formulated as LMI stability conditions where the non-smooth Lyapunov (energy) function is searched in flexible but detached regions of fuzzy state space. It has been emphasized that such flexibility is 


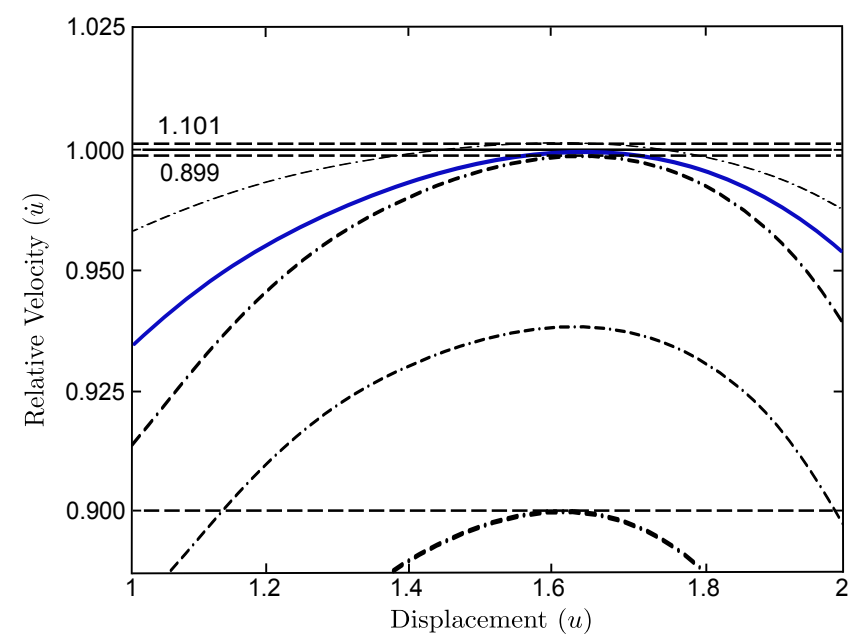

Fig. 6. An illustration of region partitioning and estimated energy levels of the non-smooth Lyapunov function candidate in the local regions (dot-dashed curves) when sliding (local) orbit grazes the switching manifold.

critical in avoiding the conservative LMI formulation should the problem be solved by interior-point convex optimization methods. A routine is further proposed for the automatic distribution of regions. Using this routine, the sliding-grazing bifurcation point can be accurately predicted. The method presented in this paper is a strong candidate for stability analysis and model-based control of the oscillator. By avoiding the complexity of discontinuity mapping, the direct numerical analysis of sliding-grazing flow and its bifurcation is now possible through low-order algorithms and existing smooth solvers.

\section{References}

[1] Filippov, A. F., and Arscott, F. M., 1988. Differential Equations with Discontinuous Righthand Sides: Control Systems, Vol. 18. Springer.

[2] Leine, R. I., and Nijmeijer, H., 2013. Dynamics and bifurcations of non-smooth mechanical systems, Vol. 18. Springer Science \& Business.

[3] Popp, K., Hinrichs, N., and Oestreich, M., 1995. "Dynamical behaviour of a friction oscillator with simultaneous self and external excitation”. Sadhana, 20(2-4), pp. 627-654.

[4] Hinrichs, N., Oestreich, M., and Popp, K., 1998. "On the modelling of friction oscillators". Journal of Sound and Vibration, 216(3), pp. 435-459.

[5] Galvanetto, U., 1999. "Nonlinear dynamics of multiple friction oscillators". Computer methods in applied mechanics and engineering, 178(3-4), pp. 291-306.

[6] Di Bernardo, M., Budd, C. J., Champneys, A. R., Kowalczyk, P., Nordmark, A. B., Tost, G. O., and Piiroinen, P. T., 2008. "Bifurcations in nonsmooth dynamical systems". SIAM review, 50(4), pp. 629-701.

[7] Galvanetto, U., 2001. "Some discontinuous bifurcations in a two-block stick-slip system". Journal of Sound and vibration, 248(4), pp. 653-669.

[8] Dankowicz, H., and Nordmark, A. B., 2000. “On the origin and bifurcations of stick-slip oscillations". Physica D: Nonlinear Phenomena, 136(3), pp. 280-302. 
[9] Di Bernardo, M., 2008. Piecewise-smooth dynamical systems: theory and applications. Springer.

[10] Leine, R., and Van Campen, D., 2002. “Discontinuous bifurcations of periodic solutions". Mathematical and computer modelling, 36(3), pp. 259-273.

[11] Pascal, M., 2008. "Dynamics of coupled oscillators excited by dry friction". Journal of Computational and Nonlinear Dynamics, 3(3), p. 031009.

[12] Nechak, L., Berger, S., and Aubry, E., 2014. "Wiener-askey and wiener-haar expansions for the analysis and prediction of limit cycle oscillations in uncertain nonlinear dynamic friction systems". Journal of Computational and Nonlinear Dynamics, 9(2), p. 011001.

[13] Di Bernardo, M., Kowalczyk, P., and Nordmark, A., 2002. "Bifurcations of dynamical systems with sliding: derivation of normal-form mappings". Physica D: Nonlinear Phenomena, 170(3), pp. 175-205.

[14] Nordmark, A. B., 1997. “Universal limit mapping in grazing bifurcations”. Physical review E, 55(1), p. 266.

[15] Kundu, S., Banerjee, S., and Giaouris, D., 2011. "Vanishing singularity in hard impacting systems". Discr. Contin. Dyn. Syst., Ser. B, 16(1), pp. 319-332.

[16] Dankowicz, H., and Jerrelind, J., 2005. "Control of near-grazing dynamics in impact oscillators". Proceedings of the Royal Society A: Mathematical, Physical and Engineering Science, 461(2063), pp. 3365-3380.

[17] Mehran, K., Zahawi, B., and Giaouris, D., 2012. "Investigation of the near-grazing behavior in hard-impact oscillators using model-based ts fuzzy approach”. Nonlinear Dynamics, 69(3), pp. 1293-1309.

[18] di Bernardo, M., Kowalczyk, P., and Nordmark, A., 2003. "Sliding bifurcations: a novel mechanism for the sudden onset of chaos in dry friction oscillators". International journal of Bifurcation and chaos, 13(10), pp. 2935-2948.

[19] Luo, A. C., and Gegg, B. C., 2006. "Periodic motions in a periodically forced oscillator moving on an oscillating belt with dry friction". Journal of computational and nonlinear dynamics, 1(3), pp. 212-220.

[20] Piiroinen, P. T., and Kuznetsov, Y. A., 2008. "An event-driven method to simulate filippov systems with accurate computing of sliding motions". ACM Transactions on Mathematical Software (TOMS), 34(3), p. 13.

[21] Mehran, K., Giaouris, D., and Zahawi, B., 2010. "Stability analysis and control of nonlinear phenomena in boost converters using model-based takagi-sugeno fuzzy approach". Circuits and Systems I: Regular Papers, IEEE Transactions on, 57(1), pp. 200-212.

[22] Tanaka, K., Ikeda, T., and Wang, H., 1998. "Fuzzy regulators and fuzzy observers: Relaxed stability conditions and lmi-based designs". IEEE Transactions on Fuzzy Systems, 6(2).

[23] Tanaka, K., 2001. Fuzzy Control Systems Design and Analysis: a Linear Matrix Inequality Approach. John Wiley \& Sons, Newark.

[24] Ting, C.-S., 2006. "Stability analysis and design of takagi-sugeno fuzzy systems". Information Sciences, 176(19), pp. 2817-2845.

[25] Narimani, M., and Lam, H.-K., 2009. "Relaxed lmi-based stability conditions for takagi-sugeno fuzzy control systems using regional-membership-function-shape-dependent analysis approach”. Fuzzy Systems, IEEE Transactions on, 17(5), pp. 1221-1228. 
[26] Dong, J., and Yang, G.-H., 2011. "Control synthesis of t-s fuzzy systems based on a new control scheme”. Fuzzy Systems, IEEE Transactions on, 19(2), pp. 323-338.

[27] Yoshitake, Y., and Sueoka, A., 2000. "Forced self-excited vibration with dry friction". Applied nonlinear dynamics and chaos of mechanical systems with discontinuities(28), pp. 237-257.

[28] Slotine, J.-J. E., Li, W., et al., 1991. Applied nonlinear control, Vol. 199. Prentice-Hall Englewood Cliffs, NJ.

[29] Boyd, S., Ghaoui, L. E., Feron, E., and Balakrishnan, V., 1994. Linear Matrix Inequalities in System and Control Theory. SIAM, Philadelphia.

[30] Khalil, H. K., and Grizzle, J., 2002. Nonlinear systems, Vol. 3. Prentice hall Upper Saddle River.

\section{List of Figures}

Fig. 1. Model of a forced oscillator with dry friction.

Fig. 2. (a) $4 T(8 \pi / \varpi)$ periodic orbit grazes the boundary of sliding region (grazing-sliding event) at $\varpi=1.7077997$ and turns to a (b) chaotic orbit. Dot-dashed line in (a) shows a stable, non-sliding periodic orbit for $\varpi=1.7082$ before sliding-grazing event, and the solid line in (a) shows the sliding orbit near the tangency of attracting switching manifold.

Fig. 3. The bifurcation diagram shows the grazing-sliding bifurcation where there is a sudden transition to a chaotic attractor.

Fig. 4. With small parameter variation, periodic (sliding) orbit $a \rightarrow b$, touches the switching manifold $\Sigma$ at $x^{*}$ undergoing a sliding-grazing bifurcation, and $b \rightarrow c$. In fact, periodic orbits $a$ and $c$ might exist for the same parameters values.

Fig. 5. Bifurcation diagram obtained from the direct simulation of the non-smooth TS fuzzy model (11),(12) shows the grazing-sliding DIB at $\varpi=1.70778$. Small figures show steady-state time responses.

Fig. 6. An illustration of region partitioning and estimated energy levels of the non-smooth Lyapunov function candidate in the local regions (dot-dashed curves) when sliding (local) orbit grazes the switching manifold. 